\title{
Fixed point and coupled fixed point theorems on $b$-metric-like spaces
}

\author{
Mohammed Ali Alghamdi ${ }^{1}$, Nawab Hussain ${ }^{1 *}$ and Peyman Salimi ${ }^{2}$
}

${ }^{*}$ Correspondence:

nhusain@kau.edu.sa

${ }^{1}$ Department of Mathematics, King Abdulaziz University, P.O. Box 80203,

Jeddah, 21589, Saudi Arabia

Full list of author information is

available at the end of the article

\begin{abstract}
We first introduce the concept of $b$-metric-like space which generalizes the notions of partial metric space, metric-like space and $b$-metric space. Then we establish the existence and uniqueness of fixed points in a $b$-metric-like space as well as in a partially ordered $b$-metric-like space. As an application, we derive some new fixed point and coupled fixed point results in partial metric spaces, metric-like spaces and $b$-metric spaces. Moreover, some examples and an application to integral equations are provided to illustrate the usability of the obtained results.
\end{abstract}

MSC: Primary 47H10; 54H25; 55M20

Keywords: partial metric space; metric-like space; $b$-metric space; $b$-metric-like space; fixed point; integral equation

\section{Introduction}

There exist many generalizations of the concept of metric spaces in the literature. In particular, Matthews [1] introduced the notion of partial metric space and proved that the Banach contraction mapping theorem can be generalized to the partial metric context for applications in program verification. After that, fixed point results in partial metric spaces have been studied by many authors $[1,2]$. The concept of $b$-metric space was introduced and studied by Bakhtin [3] and Czerwik [4]. Since then several papers have dealt with fixed point theory for single-valued and multi-valued operators in $b$-metric spaces (see [5-8] and references therein). Recently, Amini-Harandi $[9,10]$ introduced the notion of metriclike space, which is an interesting generalization of partial metric space and dislocated metric space [11-13]. In this paper, we first introduce a new generalization of metric-like space and partial metric space which is called a $b$-metric-like space. Then, we give some fixed point results in such spaces. Our fixed point theorems, even in the case of metric-like spaces and partial metric spaces, generalize and improve some well-known results in the literature. Moreover, some examples and an application to integral equations are provided to illustrate the usability of the obtained results.

\section{2 b-Metric-like spaces}

Matthews [1] introduced the concept of a partial metric space as follows.

Definition 2.1 A mapping $p: X \times X \rightarrow \mathbb{R}_{+}$, where $X$ is a nonempty set, is said to be a partial metric on $X$ if for any $x, y, z \in X$ the following four conditions hold true:

(P1) $x=y$ if and only if $p(x, x)=p(y, y)=p(x, y)$; 
(P2) $p(x, x) \leq p(x, y)$;

(P3) $p(x, y)=p(y, x)$;

(P4) $p(x, z) \leq p(x, y)+p(y, z)-p(y, y)$.

The pair $(X, p)$ is then called a partial metric space.

Definition 2.2 [9] A mapping $\sigma: X \times X \rightarrow \mathbb{R}_{+}$, where $X$ is a nonempty set, is said to be a metric-like on $X$ if for any $x, y, z \in X$ the following three conditions hold true:

$(\sigma 1) \sigma(x, y)=0 \Rightarrow x=y$;

$(\sigma 2) \sigma(x, y)=\sigma(y, x)$

$(\sigma 3) \sigma(x, z) \leq \sigma(x, y)+\sigma(y, z)$.

The pair $(X, \sigma)$ is then called a metric-like space. A metric-like on $X$ satisfies all of the conditions of a metric except that $\sigma(x, x)$ may be positive for $x \in X$.

Every partial metric space is a metric-like space but not conversely in general (see [9, 10]).

The concept of $b$-metric space was introduced by Czerwik in [4]. Since then, several papers have been published on the fixed point theory of various classes of single-valued and multi-valued operators in $b$-metric spaces (see, e.g., [6-8]).

Definition 2.3 A $b$-metric on a nonempty set $X$ is a function $\mathcal{D}: X \times X \rightarrow[0,+\infty)$ such that for all $x, y, z \in X$ and a constant $K \geq 1$ the following three conditions hold true:

$(\mathcal{D} 1)$ if $\mathcal{D}(x, y)=0 \Leftrightarrow x=y$;

(D2) $\mathcal{D}(x, y)=\mathcal{D}(y, x)$;

(D3) $\mathcal{D}(x, y) \leq K(\mathcal{D}(x, z)+\mathcal{D}(z, y))$.

The pair $(X, \mathcal{D})$ is called a $b$-metric space.

Definition 2.4 A $b$-metric-like on a nonempty set $X$ is a function $\mathcal{D}: X \times X \rightarrow[0,+\infty)$ such that for all $x, y, z \in X$ and a constant $K \geq 1$ the following three conditions hold true:

$(\mathcal{D} 1)$ if $\mathcal{D}(x, y)=0 \Rightarrow x=y$;

(D2) $\mathcal{D}(x, y)=\mathcal{D}(y, x)$;

(D3) $\mathcal{D}(x, y) \leq K(\mathcal{D}(x, z)+\mathcal{D}(z, y))$.

The pair $(X, \mathcal{D})$ is called a $b$-metric-like space.

Example 2.5 Let $X=[0, \infty)$. Define the function $\mathcal{D}: X^{2} \rightarrow[0, \infty)$ by $\mathcal{D}(x, y)=(x+y)^{2}$. Then $(X, \mathcal{D})$ is a $b$-metric-like space with constant $K=2$. Clearly, $(X, \mathcal{D})$ is not a $b$-metric or metric-like space. Indeed, for all $x, y, z \in X$,

$$
\begin{aligned}
\mathcal{D}(x, y) & =(x+y)^{2} \leq(x+z+z+y)^{2}=(x+z)^{2}+(z+y)^{2}+2(x+z)(z+y) \\
& \leq 2\left[(x+z)^{2}+(z+y)^{2}\right] \\
& =2(\mathcal{D}(x, z)+\mathcal{D}(z, y))
\end{aligned}
$$

and so $(\mathcal{D} 3)$ holds. Clearly, $(\mathcal{D} 1)$ and $(\mathcal{D} 2)$ hold. 
Similarly, we have the following example.

Example 2.6 Let $X=[0, \infty)$. Define the function $\mathcal{D}: X^{2} \rightarrow[0, \infty)$ by $\mathcal{D}(x, y)=(\max \{x$, $y\})^{2}$. Then $(X, \mathcal{D})$ is a $b$-metric-like space with constant $K=2$. Clearly, $(X, \mathcal{D})$ is not a $b$-metric or metric-like space.

Example 2.7 Let $C_{b}(X)=\left\{f: X \rightarrow \mathbb{R}: \sup _{x \in X}|f(x)|<+\infty\right\}$. The function $\mathcal{D}: X \times X \rightarrow \mathbb{R}_{+}$, defined by

$$
\mathcal{D}(f, g)=\sqrt[3]{\sup _{x \in X}(|f(x)|+|g(x)|)^{3}} \quad \text { for all } f, g \in C_{b}(X),
$$

is a $b$-metric-like with constant $K=\sqrt[3]{4}$, and so $(X, \mathcal{D}, \sqrt[3]{4})$ is a $b$-metric-like space.

For this, note that if $a, b$ are two nonnegative real numbers, then

$$
(a+b)^{3} \leq 4\left(a^{3}+b^{3}\right) \text { and } \sqrt[3]{a+b} \leq \sqrt[3]{a}+\sqrt[3]{b}
$$

This implies that

$$
\mathcal{D}(f, g) \leq \sqrt[3]{4}(\mathcal{D}(f, h)+\mathcal{D}(h, g)) \quad \text { for all } f, g, h \in C_{b}(X) .
$$

Let $(X, \mathcal{D})$ be a $b$-metric-like space. Let $x \in X$ and $r>0$, then the set

$$
B(x, r)=\{y \in X:|\mathcal{D}(x, y)-\mathcal{D}(x, x)|<r\}
$$

is called an open ball with center at $x$ and radius $r>0$.

Now we have the following definitions.

Definition 2.8 Let $(X, \mathcal{D})$ be a $b$-metric-like space, and let $\left\{x_{n}\right\}$ be a sequence of points of $X$. A point $x \in X$ is said to be the limit of the sequence $\left\{x_{n}\right\}$ if $\lim _{n \rightarrow+\infty} \mathcal{D}\left(x, x_{n}\right)=\mathcal{D}(x, x)$, and we say that the sequence $\left\{x_{n}\right\}$ is convergent to $x$ and denote it by $x_{n} \rightarrow x$ as $n \rightarrow \infty$.

Definition 2.9 Let $(X, \mathcal{D})$ be a $b$-metric-like space.

(S1) A sequence $\left\{x_{n}\right\}$ is called Cauchy if and only if $\lim _{m, n \rightarrow \infty} \mathcal{D}\left(x_{n}, x_{m}\right)$ exists and is finite.

(S2) A $b$-metric-like space $(X, \mathcal{D})$ is said to be complete if and only if every Cauchy sequence $\left\{x_{n}\right\}$ in $\mathrm{X}$ converges to $x \in X$ so that

$$
\lim _{m, n \rightarrow \infty} \mathcal{D}\left(x_{n}, x_{m}\right)=\mathcal{D}(x, x)=\lim _{n \rightarrow \infty} \mathcal{D}\left(x_{n}, x\right)
$$

Proposition 2.10 Let $(X, \mathcal{D}, K)$ be a b-metric-like space, and let $\left\{x_{n}\right\}$ be a sequence in $X$ such that $\lim _{n \rightarrow \infty} \mathcal{D}\left(x_{n}, x\right)=0$. Then

(A) $x$ is unique;

(B) $\frac{1}{K} \mathcal{D}(x, y) \leq \lim _{n \rightarrow \infty} \mathcal{D}\left(x_{n}, y\right) \leq K \mathcal{D}(x, y)$ for all $y \in X$. 
Proof Let us prove (A).

Assume that there exists a $y \in X$ such that $\lim _{n \rightarrow \infty} \mathcal{D}\left(x_{n}, y\right)=0$, then

$$
0 \leq \mathcal{D}(y, x) \leq K\left(\lim _{n \rightarrow \infty} \mathcal{D}\left(x_{n}, y\right)+\lim _{n \rightarrow \infty} \mathcal{D}\left(x_{n}, x\right)\right)=0 .
$$

Hence, from $(\mathcal{D} 1)$ we have $y=x$.

(B)

From (D3) we have

$$
\frac{1}{K} \mathcal{D}(x, y)-\lim _{n \rightarrow \infty} \mathcal{D}\left(x_{n}, x\right) \leq \lim _{n \rightarrow \infty} \mathcal{D}\left(x_{n}, y\right) \leq K\left(\mathcal{D}(x, y)+\lim _{n \rightarrow \infty} \mathcal{D}\left(x_{n}, x\right)\right)
$$

and so

$$
\frac{1}{K} \mathcal{D}(x, y) \leq \lim _{n \rightarrow \infty} \mathcal{D}\left(x_{n}, y\right) \leq K \mathcal{D}(x, y) \quad \text { for all } y \in X .
$$

Definition 2.11 Let $(X, \mathcal{D})$ be a $b$-metric-like space, and let $U$ be a subset of $X$. We say $U$ is an open subset of $X$ if for all $x \in U$ there exists $r>0$ such that $B(x, r) \subseteq U$. Also, $V \subseteq X$ is a closed subset of $X$ if $X \backslash V$ is an open subset of $X$.

Proposition 2.12 Let $(X, \mathcal{D}, K)$ be a b-metric-like space, and let $V$ be a subset of $X$. Then $V$ is closed if and only if for any sequence $\left\{x_{n}\right\}$ in $V$, which converges to $x$, we have $x \in V$.

Proof At first, we suppose that $V$ is closed. Let $x \notin V$. By the above definition, $X \backslash V$ is an open set. Then there is an $r>0$ such that $B(x, r) \subseteq X \backslash V$. On the other hand, since $x_{n} \rightarrow x$ as $n \rightarrow \infty$, then

$$
\lim _{n \rightarrow \infty}\left|\mathcal{D}\left(x_{n}, x\right)-\mathcal{D}(x, x)\right|=0
$$

Hence, there exists $n_{0} \in \mathbb{N}$ such that for all $n \geq n_{0}$ we have

$$
\left|\mathcal{D}\left(x_{n}, x\right)-\mathcal{D}(x, x)\right|<r
$$

That is, for all $n \geq n_{0},\left\{x_{n}\right\} \subseteq B(x, r) \subseteq X \backslash V$, which is a contradiction. Since for all $n \in \mathbb{N}$, $\left\{x_{n}\right\} \subseteq V$. Conversely, suppose that for any sequence $\left\{x_{n}\right\}$ in $V$ which converges to $x$, we have $x \in V$. Let $y \notin V$. Let us prove that there exists $r_{0}>0$ such that $B\left(y, r_{0}\right) \cap V=\emptyset$. Assume to the contrary that for all $r>0$, we have $B(y, r) \cap V \neq \emptyset$. Then, for all $n \in \mathbb{N}$, chose $x_{n} \in B(y, 1 / n) \cap V \neq \emptyset$. Therefore, $\left|\mathcal{D}\left(x_{n}, y\right)-\mathcal{D}(y, y)\right|<1 / n$ for all $n \in \mathbb{N}$. Hence, $x_{n} \rightarrow y$ as $n \rightarrow \infty$. Our assumption on $V$ implies $y \in V$, which is a contradiction. Then, for all $y \notin V$, there exists $r_{0}>0$ such that $B\left(y, r_{0}\right) \cap V=\emptyset$. That is, $V$ is closed.

Lemma 2.13 Let $(X, \mathcal{D}, K)$ be a b-metric-like space, and let $\left\{x_{k}\right\}_{k=0}^{n} \subset X$. Then

$$
\mathcal{D}\left(x_{n}, x_{0}\right) \leq K \mathcal{D}\left(x_{0}, x_{1}\right)+\cdots+K^{n-1} \mathcal{D}\left(x_{n-2}, x_{n-1}\right)+K^{n-1} \mathcal{D}\left(x_{n-1}, x_{n}\right)
$$

From Lemma 2.13, we deduce the following result. 
Lemma 2.14 Let $\left\{y_{n}\right\}$ be a sequence in a $b$-metric-like space $(X, \mathcal{D}, K)$ such that

$$
\mathcal{D}\left(y_{n}, y_{n+1}\right) \leq \lambda \mathcal{D}\left(y_{n-1}, y_{n}\right)
$$

for some $\lambda, 0<\lambda<1 / K$, and each $n \in \mathbb{N}$. Then $\lim _{m, n \rightarrow \infty} \mathcal{D}\left(y_{m}, y_{n}\right)=0$.

Let $(X, \mathcal{D}, K)$ be a $b$-metric-like space. Define $\mathcal{D}^{s}: X^{2} \rightarrow[0, \infty)$ by

$$
\mathcal{D}^{s}(x, y)=|2 \mathcal{D}(x, y)-\mathcal{D}(x, x)-\mathcal{D}(y, y)|
$$

Clearly, $\mathcal{D}^{s}(x, x)=0$ for all $x \in X$.

\section{Fixed point results for expansive mappings}

The study of expansive mappings is a very interesting research area in fixed point theory (see, e.g., [14-21]). In this section we prove some new fixed point results on expansive mappings in the setting of a $b$-metric-like space. Our results generalize and extend some old and recent fixed point results in the literature.

Theorem 3.1 Let $(X, \mathcal{D}, K)$ be a complete b-metric-like space. Assume that the mapping $T: X \rightarrow X$ is onto and satisfies

$$
\mathcal{D}(T x, T y) \geq\left[R+L \min \left\{\mathcal{D}^{s}(x, T x), \mathcal{D}^{s}(y, T y), \mathcal{D}^{s}(x, T y), \mathcal{D}^{s}(y, T x)\right\}\right] \mathcal{D}(x, y)
$$

for all $x, y \in X$, where $R>K, L \geq 0$. Then $T$ has a fixed point.

Proof Let $x_{0} \in X$, since $T$ is onto, then there exists $x_{1} \in X$ such that $x_{0}=T x_{1}$. By continuing this process, we get $x_{n}=T x_{n+1}$ for all $n \in \mathbb{N} \cup\{0\}$. In case $x_{n_{0}}=x_{n_{0}+1}$ for some $n_{0} \in \mathbb{N} \cup\{0\}$, then it is clear that $x_{n_{0}}$ is a fixed point of $T$. Now assume that $x_{n} \neq x_{n+1}$ for all $n$. From (3.1) with $x=x_{n}$ and $y=x_{n+1}$ we get

$$
\begin{aligned}
\mathcal{D}\left(T x_{n}, T x_{n+1}\right) \geq & {\left[R+L \min \left\{\mathcal{D}^{s}\left(x_{n}, T x_{n}\right), \mathcal{D}^{s}\left(x_{n+1}, T x_{n+1}\right),\right.\right.} \\
& \left.\left.\mathcal{D}^{s}\left(x_{n}, T x_{n+1}\right), \mathcal{D}^{s}\left(x_{n+1}, T x_{n}\right)\right\}\right] \mathcal{D}\left(x_{n}, x_{n+1}\right),
\end{aligned}
$$

which implies

$$
\begin{aligned}
\mathcal{D}\left(x_{n-1}, x_{n}\right) \geq & {\left[R+L \min \left\{\mathcal{D}^{s}\left(x_{n}, x_{n-1}\right), \mathcal{D}^{s}\left(x_{n+1}, x_{n}\right),\right.\right.} \\
& \left.\left.\mathcal{D}^{s}\left(x_{n}, x_{n}\right), \mathcal{D}^{s}\left(x_{n+1}, x_{n-1}\right)\right\}\right] \mathcal{D}\left(x_{n}, x_{n+1}\right)=R \mathcal{D}\left(x_{n}, x_{n+1}\right),
\end{aligned}
$$

and so

$$
\mathcal{D}\left(x_{n}, x_{n+1}\right) \leq h \mathcal{D}\left(x_{n-1}, x_{n}\right), \quad \text { where } h=\frac{1}{R}<\frac{1}{K} .
$$

Then by Lemma 2.14 we have $\lim _{m, n \rightarrow \infty} \mathcal{D}\left(x_{n}, x_{m}\right)=0$. Now, since $\lim _{m, n \rightarrow \infty} \mathcal{D}\left(x_{n}, x_{m}\right)=0$ exists (and is finite), so $\left\{x_{n}\right\}$ is a Cauchy sequence. Since $(X, \mathcal{D}, K)$ is a complete $b$-metriclike space, the sequence $\left\{x_{n}\right\}$ in $X$ converges to $z \in X$ so that

$$
\lim _{m, n \rightarrow \infty} \mathcal{D}\left(x_{n}, z\right)=\mathcal{D}(z, z)=\lim _{m, n \rightarrow \infty} \mathcal{D}\left(x_{n}, x_{m}\right)=0 .
$$


Since $T$ is onto, there exists $w \in X$ such that $z=T w$. From (3.1) we have

$$
\begin{aligned}
\mathcal{D}\left(x_{n}, z\right)= & \mathcal{D}\left(T x_{n+1}, T w\right) \\
\geq & {\left[R+L \min \left\{\mathcal{D}^{s}\left(x_{n+1}, T x_{n+1}\right), \mathcal{D}^{s}(w, T w),\right.\right.} \\
& \left.\left.\mathcal{D}^{s}\left(x_{n+1}, T w\right), \mathcal{D}^{s}\left(w, T x_{n+1}\right)\right\}\right] \mathcal{D}\left(x_{n+1}, w\right) \\
= & {\left[R+L \min \left\{\mathcal{D}^{s}\left(x_{n+1}, x_{n}\right), \mathcal{D}^{s}(w, z),\right.\right.} \\
& \left.\left.\mathcal{D}^{s}\left(x_{n+1}, z\right), \mathcal{D}^{s}\left(w, x_{n}\right)\right\}\right] \mathcal{D}\left(x_{n+1}, w\right) .
\end{aligned}
$$

Taking limit as $n \rightarrow \infty$ in the above inequality, we get

$$
0=\lim _{n \rightarrow \infty} \mathcal{D}\left(x_{n}, z\right) \geq R \lim _{n \rightarrow \infty} \mathcal{D}\left(x_{n+1}, w\right)
$$

which implies $\lim _{n \rightarrow \infty} \mathcal{D}\left(x_{n+1}, w\right)=0$. By Proposition $2.10(\mathrm{~A})$, we get $z=w$. That is, $z=$ $T z$.

If in Theorem 3.1 we take $L=0$, then we deduce the following corollary.

Corollary 3.2 Let $(X, \mathcal{D}, K)$ be a complete b-metric-like space. Assume that the mapping $T: X \rightarrow X$ is onto and satisfies

$$
\mathcal{D}(T x, T y) \geq R \mathcal{D}(x, y)
$$

for all $x, y \in X$, where $R>K$. Then $T$ has a fixed point.

Example 3.3 Let $X=[0, \infty)$ and let a $b$-metric-like $\mathcal{D}: X \times X \rightarrow \mathbb{R}_{+}$be defined by

$$
\mathcal{D}(x, y)=(x+y)^{2}
$$

Clearly, $(X, \mathcal{D}, 2)$ is a complete $b$-metric-like space. Let $T: X \rightarrow X$ be defined by

$$
T x= \begin{cases}6 x & \text { if } x \in[0,1) \\ 5 x+1 & \text { if } x \in[1,2) \\ 4 x+3 & \text { if } x \in[2, \infty)\end{cases}
$$

Also, clearly, $T$ is an onto mapping. Now, we consider following cases:

Let $x, y \in[0,1)$, then

$$
\mathcal{D}(T x, T y)=(6 x+6 y)^{2}=36(x+y)^{2} \geq 3(x+y)^{2}=3 \mathcal{D}(x, y) .
$$

Let $x, y \in[1,2)$, then

$$
\mathcal{D}(T x, T y)=(5 x+5 y+2)^{2} \geq(5 x+5 y)^{2}=25(x+y)^{2} \geq 3(x+y)^{2}=3 \mathcal{D}(x, y) .
$$


Let $x, y \in[2, \infty)$, then

$$
\mathcal{D}(T x, T y)=(4 x+4 y+6)^{2} \geq(4 x+4 y)^{2}=16(x+y)^{2} \geq 3(x+y)^{2}=3 \mathcal{D}(x, y)
$$

Let $x \in[0,1)$ and $y \in[1,2)$, then

$$
\mathcal{D}(T x, T y)=(6 x+5 y+1)^{2} \geq(5 x+5 y)^{2}=25(x+y)^{2} \geq 3(x+y)^{2}=3 \mathcal{D}(x, y) .
$$

Let $x \in[0,1)$ and $y \in[2, \infty)$, then

$$
\mathcal{D}(T x, T y)=(6 x+4 y+3)^{2} \geq(4 x+4 y)^{2}=16(x+y)^{2} \geq 3(x+y)^{2}=3 \mathcal{D}(x, y) .
$$

Let $x \in[1,2)$ and $y \in[2, \infty)$, then

$$
\mathcal{D}(T x, T y)=(5 x+4 y+4)^{2} \geq(4 x+4 y)^{2}=16(x+y)^{2} \geq 3(x+y)^{2}=3 \mathcal{D}(x, y) .
$$

That is, $\mathcal{D}(T x, T y) \geq R \mathcal{D}(x, y)$ for all $x, y \in X$, where $R=3>2=K$. The conditions of Corollary 3.2 are satisfied and $T$ has a fixed point $x=0$.

Let $\Psi_{\mathcal{B}}^{L}$ denote the class of those functions $\mathcal{B}:(0, \infty) \rightarrow\left(L^{2}, \infty\right)$ which satisfy the condition $\mathcal{B}\left(t_{n}\right) \rightarrow\left(L^{2}\right)^{+} \Rightarrow t_{n} \rightarrow 0$, where $L>0$.

Theorem 3.4 Let $(X, \mathcal{D}, K)$ be a complete b-metric-like space. Assume that the mapping $T: X \rightarrow X$ is onto and satisfies

$$
\mathcal{D}(T x, T y) \geq \mathcal{B}(\mathcal{D}(x, y)) \mathcal{D}(x, y)
$$

for all $x, y \in X$, where $\mathcal{B} \in \Psi_{\mathcal{B}}^{K}$. Then $T$ has a fixed point.

Proof Let $x_{0} \in X$, since $T$ is onto, so there exists $x_{1} \in X$ such that $x_{0}=T x_{1}$. By continuing this process, we get $x_{n}=T x_{n+1}$ for all $n \in \mathbb{N} \cup\{0\}$. In case $x_{n_{0}}=x_{n_{0}+1}$ for some $n_{0} \in \mathbb{N} \cup\{0\}$, then it is clear that $x_{n_{0}}$ is a fixed point of $T$. Now assume that $x_{n} \neq x_{n+1}$ for all $n$. From (3.2) with $x=x_{n}$ and $y=x_{n+1}$, we get

$$
\begin{aligned}
\mathcal{D}\left(x_{n-1}, x_{n}\right) & =\mathcal{D}\left(T x_{n}, T x_{n+1}\right) \geq \mathcal{B}\left(\mathcal{D}\left(x_{n}, x_{n+1}\right)\right) \mathcal{D}\left(x_{n}, x_{n+1}\right) \\
& \geq K^{2} \mathcal{D}\left(x_{n}, x_{n+1}\right) \geq \mathcal{D}\left(x_{n}, x_{n+1}\right)
\end{aligned}
$$

Then the sequence $\left\{\mathcal{D}\left(x_{n}, x_{n+1}\right)\right\}$ is a decreasing sequence in $\mathbb{R}_{+}$and so there exists $s \geq 0$ such that $\lim _{n \rightarrow \infty} \mathcal{D}\left(x_{n}, x_{n+1}\right)=s$. Let us prove that $s=0$. Suppose to the contrary that $s>0$. By (3.3) we can deduce

$$
K^{2} \frac{\mathcal{D}\left(x_{n-1}, x_{n}\right)}{\mathcal{D}\left(x_{n}, x_{n+1}\right)} \geq \frac{\mathcal{D}\left(x_{n-1}, x_{n}\right)}{\mathcal{D}\left(x_{n}, x_{n+1}\right)} \geq \mathcal{B}\left(\mathcal{D}\left(x_{n}, x_{n+1}\right)\right) \geq K^{2}
$$

By taking limit as $n \rightarrow \infty$ in the above inequality, we have $\lim _{n \rightarrow \infty} \mathcal{B}\left(\mathcal{D}\left(x_{n}, x_{n+1}\right)\right)=K^{2}$. Hence,

$$
s=\lim _{n \rightarrow \infty} \mathcal{D}\left(x_{n}, x_{n+1}\right)=0,
$$


which is a contradiction. That is, $s=0$. We shall show that $\lim _{\sup _{m, n \rightarrow \infty}} \mathcal{D}\left(x_{n}, x_{m}\right)=0$. Suppose to the contrary that

$$
\limsup _{m, n \rightarrow \infty} \mathcal{D}\left(x_{n}, x_{m}\right)>0
$$

By (3.2) we have

$$
\mathcal{D}\left(x_{n}, x_{m}\right)=\mathcal{D}\left(T x_{n+1}, T x_{m+1}\right) \geq \mathcal{B}\left(\mathcal{D}\left(x_{n+1}, x_{m+1}\right)\right) \mathcal{D}\left(x_{n+1}, x_{m+1}\right) .
$$

That is,

$$
\frac{\mathcal{D}\left(x_{n}, x_{m}\right)}{\mathcal{B}\left(\mathcal{D}\left(x_{n+1}, x_{m+1}\right)\right)} \geq \mathcal{D}\left(x_{n+1}, x_{m+1}\right)
$$

Then by $(\mathcal{D} 3)$ we get

$$
\begin{aligned}
\mathcal{D}\left(x_{n}, x_{m}\right) & \leq K \mathcal{D}\left(x_{n}, x_{n+1}\right)+K^{2} \mathcal{D}\left(x_{n+1}, x_{m+1}\right)+K^{2} \mathcal{D}\left(x_{m+1}, x_{m}\right) \\
& \leq K \mathcal{D}\left(x_{n}, x_{n+1}\right)+K^{2} \frac{\mathcal{D}\left(x_{n}, x_{m}\right)}{\mathcal{B}\left(\mathcal{D}\left(x_{n+1}, x_{m+1}\right)\right)}+K^{2} \mathcal{D}\left(x_{m+1}, x_{m}\right) .
\end{aligned}
$$

Therefore,

$$
\mathcal{D}\left(x_{n}, x_{m}\right) \leq\left(1-\frac{K^{2}}{\mathcal{B}\left(\mathcal{D}\left(x_{n+1}, x_{m+1}\right)\right)}\right)^{-1}\left(K \mathcal{D}\left(x_{n}, x_{n+1}\right)+K^{2} \mathcal{D}\left(x_{m+1}, x_{m}\right)\right) .
$$

By taking limit as $m, n \rightarrow \infty$ in the above inequality, since $\limsup _{m, n \rightarrow \infty} \mathcal{D}\left(x_{n}, x_{m}\right)>0$ and $s=\lim _{n \rightarrow \infty} \mathcal{D}\left(x_{n}, x_{n+1}\right)=0$, then we obtain

$$
\limsup _{m, n \rightarrow \infty}\left(1-\frac{K^{2}}{\mathcal{B}\left(\mathcal{D}\left(x_{n+1}, x_{m+1}\right)\right)}\right)^{-1}=\infty
$$

which implies

$$
\limsup _{m, n \rightarrow \infty} \mathcal{B}\left(\mathcal{D}\left(x_{n+1}, x_{m+1}\right)\right)=\left(K^{2}\right)^{+},
$$

and so

$$
\limsup _{m, n \rightarrow \infty} \mathcal{D}\left(x_{n+1}, x_{m+1}\right)=0,
$$

which is a contradiction. Hence, $\limsup _{m, n \rightarrow \infty} \mathcal{D}\left(x_{n}, x_{m}\right)=0$. Now, since $\lim _{m, n \rightarrow \infty} \mathcal{D}\left(x_{n}\right.$, $\left.x_{m}\right)=0$ exists (and is finite), so $\left\{x_{n}\right\}$ is a Cauchy sequence. Since $(X, \mathcal{D}, K)$ is a complete $b$-metric-like space, the sequence $\left\{x_{n}\right\}$ in $\mathrm{X}$ converges to $z \in X$ so that

$$
\lim _{m, n \rightarrow \infty} \mathcal{D}\left(x_{n}, z\right)=\mathcal{D}(z, z)=\lim _{m, n \rightarrow \infty} \mathcal{D}\left(x_{n}, x_{m}\right)=0 .
$$

As $T$ is onto, so there exists $w \in X$ such that $z=T w$. Let us prove that $w=z$. Suppose to the contrary that $z \neq w$. Then by (3.2) we have

$$
\mathcal{D}\left(x_{n}, z\right)=\mathcal{D}\left(T x_{n+1}, T w\right) \geq \mathcal{B}\left(\mathcal{D}\left(x_{n+1}, w\right)\right) \mathcal{D}\left(x_{n+1}, w\right) .
$$


By taking limit as $n \rightarrow \infty$ in the above inequality and applying Proposition 2.10(B), we have

$$
0=\lim _{n \rightarrow \infty} \mathcal{D}\left(x_{n}, z\right) \geq \lim _{n \rightarrow \infty} \mathcal{B}\left(\mathcal{D}\left(x_{n+1}, w\right)\right) \lim _{n \rightarrow \infty} \mathcal{D}\left(x_{n+1}, w\right) \geq \frac{1}{K} \lim _{n \rightarrow \infty} \mathcal{B}\left(\mathcal{D}\left(x_{n+1}, z\right)\right) \mathcal{D}(z, w)
$$

and hence

$$
\lim _{n \rightarrow \infty} \mathcal{B}\left(\mathcal{D}\left(x_{n+1}, z\right)\right)=0
$$

which is a contradiction. Indeed, $\lim _{n \rightarrow \infty} \mathcal{B}\left(\mathcal{D}\left(x_{n+1}, z\right)\right) \geq K^{2}$. Since $\mathcal{B}(t)>K^{2}$ for all $t \in$ $[0, \infty)$, therefore $z=w$. That is, $z=T w=T z$.

Example 3.5 Let $X=[0, \infty)$ and $\mathcal{D}: X \times X \rightarrow \mathbb{R}_{+}$be defined by

$$
\mathcal{D}(x, y)=(\max \{x, y\})^{2} .
$$

Clearly, $(X, \mathcal{D}, 2)$ is a complete $b$-metric-like space. Let $T: X \rightarrow X$ be defined by

$$
T x=4 x \sqrt{1+x^{2}} .
$$

Also define $\mathcal{B}:(0, \infty) \rightarrow(4, \infty)$ by $\mathcal{B}(t)=4(1+t)$. At first we show that $T$ is an onto mapping. For a given $a \in X$, we choose $x_{0}=\frac{1}{2} \sqrt{\sqrt{4+a^{2}}-2}$. Then

$$
T x_{0}=\left(\sqrt{\sqrt{4+a^{2}}-2}\right)\left(\sqrt{\sqrt{4+a^{2}}+2}\right)=a \text {. }
$$

So, $T$ is an onto mapping. Without loss of generality, we assume that $x \leq y$. Now, since

$$
T y \geq 2 y \sqrt{1+y^{2}},
$$

So

$$
(T y)^{2} \geq 4\left(1+y^{2}\right) y^{2}
$$

equivalently,

$$
(\max \{T x, T y\})^{2} \geq 4\left(1+(\max \{x, y\})^{2}\right)(\max \{x, y\})^{2}
$$

and hence

$$
\mathcal{D}(T x, T y) \geq 4(1+\mathcal{D}(x, y)) \mathcal{D}(x, y) .
$$

That is,

$$
\mathcal{D}(T x, T y) \geq \mathcal{B}(\mathcal{D}(x, y)) \mathcal{D}(x, y) .
$$

The conditions of Theorem 3.4 hold and $T$ has a fixed point (here, $x=0$ is a fixed point of $T)$. 
Note that $b$-metric-like spaces are a proper extension of partial metric, metric-like and $b$-metric spaces. Hence, we can deduce the following corollaries in the settings of partial metric, metric-like and $b$-metric spaces, respectively.

Corollary 3.6 Let $(X, p)$ be a complete partial metric space. Assume that the mapping $T: X \rightarrow X$ is onto and satisfies

$$
p(T x, T y) \geq \mathcal{B}(p(x, y)) p(x, y)
$$

for all $x, y \in X$, where $\mathcal{B} \in \Psi_{\mathcal{B}}^{1}$. Then $T$ has a fixed point.

Corollary 3.7 Let $(X, \sigma)$ be a complete metric-like space. Assume that the mapping $T$ : $X \rightarrow X$ is onto and satisfies

$$
\sigma(T x, T y) \geq \mathcal{B}(\sigma(x, y)) \sigma(x, y)
$$

for all $x, y \in X$, where $\mathcal{B} \in \Psi_{\mathcal{B}}^{1}$. Then $T$ has a fixed point.

Corollary 3.8 Let $(X, d, K)$ be a complete $b$-metric space. Assume that the mapping $T$ : $X \rightarrow X$ is onto and satisfies

$$
d(T x, T y) \geq \mathcal{B}(d(x, y)) d(x, y)
$$

for all $x, y \in X$, where $\mathcal{B} \in \Psi_{\mathcal{B}}^{K}$. Then $T$ has a fixed point.

\section{Fixed point results in partially ordered $b$-metric-like spaces}

In this section we prove certain new fixed point theorems in partially ordered $b$-metriclike spaces which generalize and extend corresponding results of Amini-Harandi [9, 10] and many others (see [22]).

Let $\Psi_{\mathcal{L}}^{L}$ denote the class of those functions $\mathcal{L}:(0, \infty) \rightarrow\left(0, \frac{1}{L^{2}}\right)$ which satisfy the condition $\mathcal{L}\left(t_{n}\right) \rightarrow\left(\frac{1}{L^{2}}\right)^{+} \Rightarrow t_{n} \rightarrow 0$, where $L>0$.

Theorem 4.1 Let $(X, \mathcal{D}, K, \preceq)$ be a partially ordered complete b-metric-like space, and let $T: X \rightarrow X$ be a non-decreasing mapping such that

$$
\mathcal{D}(T x, T y) \leq \mathcal{L}(M(x, y)) M(x, y)+\mathcal{J}(N(x, y)) N(x, y)
$$

for all $x, y \in X$ with $x \preceq y$, where $\mathcal{L} \in \Psi_{\mathcal{L}}^{K}, \mathcal{J}:[0, \infty) \rightarrow[0, \infty)$ is a bounded function and

$$
M(x, y)=\max \left\{\mathcal{D}(x, y), \mathcal{D}(x, T x), \mathcal{D}(y, T y), \frac{\mathcal{D}(x, T y)+\mathcal{D}(y, T x)}{6 K}\right\}
$$

and

$$
N(x, y)=\min \left\{\mathcal{D}^{s}(x, T x), \mathcal{D}^{s}(y, T y), \mathcal{D}^{s}(x, T y), \mathcal{D}^{s}(y, T x)\right\}
$$


(i) there exists $x_{0} \in X$ such that $x_{0} \preceq T x_{0}$;

(ii) for an increasing sequence $\left\{x_{n}\right\} \subset X$ converging to $x \in X$, we have $x_{n} \preceq x$ for all $n \in \mathbb{N}$; then $T$ has a fixed point.

Proof Let $x_{0} \preceq T x_{0}$. If $x_{0}=T x_{0}$, then the result is proved. Hence we suppose that $x_{0} \prec f x_{0}$. Define a sequence $\left\{x_{n}\right\}$ by $x_{n}=T^{n} x_{0}=T x_{n-1}$ for all $n \in \mathbb{N}$. Since $T$ is non-decreasing and $x_{0} \prec T x_{0}$, then

$$
x_{0} \prec x_{1} \preceq x_{2} \preceq \cdots,
$$

and hence $\left\{x_{n}\right\}$ is a non-decreasing sequence. If $x_{n}=x_{n+1}=T x_{n}$ for some $n \in \mathbb{N}$, then the result is proved as $x_{n}$ is a fixed point of $T$. In what follows we will suppose that $x_{n} \neq x_{n+1}$ for all $n \in \mathbb{N}$. From (4.1) and (4.2) we have

$$
\begin{aligned}
\mathcal{D}\left(x_{n}, x_{n+1}\right) & =\mathcal{D}\left(T x_{n-1}, T x_{n}\right) \\
& \leq \mathcal{L}\left(M\left(x_{n-1}, x_{n}\right)\right) M\left(x_{n-1}, x_{n}\right)+\mathcal{J}\left(N\left(x_{n-1}, x_{n}\right)\right) N\left(x_{n-1}, x_{n}\right),
\end{aligned}
$$

where

$$
\begin{aligned}
N\left(x_{n-1}, x_{n}\right) & =\min \left\{\mathcal{D}^{s}\left(x_{n-1}, T x_{n-1}\right), \mathcal{D}^{s}\left(x_{n}, T x_{n}\right), \mathcal{D}^{s}\left(x_{n-1}, T x_{n}\right), \mathcal{D}^{s}\left(x_{n}, T x_{n-1}\right)\right\} \\
& =\min \left\{\mathcal{D}^{s}\left(x_{n-1}, x_{n}\right), \mathcal{D}^{s}\left(x_{n}, x_{n+1}\right), \mathcal{D}^{s}\left(x_{n-1}, x_{n+1}\right), \mathcal{D}^{s}\left(x_{n}, x_{n}\right)\right\}=0 .
\end{aligned}
$$

Then

$$
\mathcal{D}\left(x_{n}, x_{n+1}\right) \leq \mathcal{L}\left(M\left(x_{n-1}, x_{n}\right)\right) M\left(x_{n-1}, x_{n}\right)
$$

On the other hand, from $(\mathcal{D} 3)$ we have

$$
\mathcal{D}\left(x_{n-1}, x_{n+1}\right) \leq K\left(\mathcal{D}\left(x_{n}, x_{n-1}\right)+\mathcal{D}\left(x_{n}, x_{n+1}\right)\right)
$$

and

$$
\mathcal{D}\left(x_{n}, x_{n}\right) \leq 2 K \mathcal{D}\left(x_{n}, x_{n+1}\right) \leq 2 K\left(\mathcal{D}\left(x_{n}, x_{n-1}\right)+\mathcal{D}\left(x_{n}, x_{n+1}\right)\right)
$$

Then

$$
\frac{\mathcal{D}\left(x_{n-1}, x_{n+1}\right)+\mathcal{D}\left(x_{n}, x_{n}\right)}{6 K} \leq \frac{1}{2}\left(\mathcal{D}\left(x_{n}, x_{n-1}\right)+\mathcal{D}\left(x_{n}, x_{n+1}\right)\right)
$$

and hence

$$
\begin{aligned}
& M\left(x_{n-1}, x_{n}\right) \\
& \quad=\max \left\{\mathcal{D}\left(x_{n-1}, x_{n}\right), \mathcal{D}\left(x_{n-1}, T x_{n-1}\right), \mathcal{D}\left(x_{n}, T x_{n}\right), \frac{\mathcal{D}\left(x_{n-1}, T x_{n}\right)+\mathcal{D}\left(x_{n}, T x_{n-1}\right)}{6 K}\right\} \\
& \quad=\max \left\{\mathcal{D}\left(x_{n-1}, x_{n}\right), \mathcal{D}\left(x_{n}, x_{n+1}\right), \frac{\mathcal{D}\left(x_{n-1}, x_{n+1}\right)+\mathcal{D}\left(x_{n}, x_{n}\right)}{6 K}\right\}
\end{aligned}
$$




$$
\begin{aligned}
& \leq \max \left\{\mathcal{D}\left(x_{n-1}, x_{n}\right), \mathcal{D}\left(x_{n}, x_{n+1}\right), \frac{1}{2}\left(\mathcal{D}\left(x_{n}, x_{n-1}\right)+\mathcal{D}\left(x_{n}, x_{n+1}\right)\right)\right\} \\
& =\max \left\{\mathcal{D}\left(x_{n-1}, x_{n}\right), \mathcal{D}\left(x_{n}, x_{n+1}\right)\right\} \leq M\left(x_{n-1}, x_{n}\right) .
\end{aligned}
$$

That is,

$$
M\left(x_{n-1}, x_{n}\right)=\max \left\{\mathcal{D}\left(x_{n-1}, x_{n}\right), \mathcal{D}\left(x_{n}, x_{n+1}\right)\right\}
$$

Now by (4.3) we get

$$
\mathcal{D}\left(x_{n}, x_{n+1}\right) \leq \mathcal{L}\left(\max \left\{\mathcal{D}\left(x_{n-1}, x_{n}\right), \mathcal{D}\left(x_{n}, x_{n+1}\right)\right\}\right) \max \left\{\mathcal{D}\left(x_{n-1}, x_{n}\right), \mathcal{D}\left(x_{n}, x_{n+1}\right)\right\}
$$

If $\max \left\{\mathcal{D}\left(x_{n-1}, x_{n}\right), \mathcal{D}\left(x_{n}, x_{n+1}\right)\right\}=\mathcal{D}\left(x_{n}, x_{n+1}\right)$, then

$$
\mathcal{D}\left(x_{n}, x_{n+1}\right) \leq \mathcal{L}\left(\mathcal{D}\left(x_{n}, x_{n+1}\right)\right) \mathcal{D}\left(x_{n}, x_{n+1}\right)<\frac{1}{K^{2}} \mathcal{D}\left(x_{n}, x_{n+1}\right) \leq \mathcal{D}\left(x_{n}, x_{n+1}\right),
$$

which is a contradiction. Hence,

$$
\mathcal{D}\left(x_{n}, x_{n+1}\right) \leq \mathcal{L}\left(\mathcal{D}\left(x_{n-1}, x_{n}\right)\right) \mathcal{D}\left(x_{n-1}, x_{n}\right) \leq \mathcal{D}\left(x_{n-1}, x_{n}\right),
$$

and so the sequence $\left\{\mathcal{D}\left(x_{n}, x_{n+1}\right)\right\}$ is a decreasing sequence in $\mathbb{R}_{+}$. Then there exists $s \geq 0$ such that $\lim _{n \rightarrow \infty} \mathcal{D}\left(x_{n}, x_{n+1}\right)=s$. By (4.4) we can write

$$
\frac{\mathcal{D}\left(x_{n}, x_{n+1}\right)}{K^{2} \mathcal{D}\left(x_{n-1}, x_{n}\right)} \leq \frac{\mathcal{D}\left(x_{n}, x_{n+1}\right)}{\mathcal{D}\left(x_{n-1}, x_{n}\right)} \leq \mathcal{L}\left(\mathcal{D}\left(x_{n-1}, x_{n}\right)\right) \leq \frac{1}{K^{2}}
$$

Taking limit as $n \rightarrow \infty$ in the above inequality, we get

$$
\lim _{n \rightarrow \infty} \mathcal{L}\left(\mathcal{D}\left(x_{n-1}, x_{n}\right)\right)=\frac{1}{K^{2}}
$$

and so $s=\lim _{n \rightarrow \infty} \mathcal{D}\left(x_{n-1}, x_{n}\right)=0$. Now we want to show that $\limsup _{m, n \rightarrow \infty} \mathcal{D}\left(x_{n}, x_{m}\right)=0$. Suppose to the contrary that

$$
\limsup _{m, n \rightarrow \infty} \mathcal{D}\left(x_{n}, x_{m}\right)>0 \text {. }
$$

At first,

$$
\begin{aligned}
\limsup _{m, n \rightarrow \infty} N\left(x_{n}, x_{m}\right)= & \limsup _{m, n \rightarrow \infty} \min \left\{\mathcal{D}^{s}\left(x_{n}, x_{n+1}\right), \mathcal{D}^{s}\left(x_{m}, x_{m+1}\right),\right. \\
& \left.\mathcal{D}^{s}\left(x_{n}, x_{m+1}\right), \mathcal{D}^{s}\left(x_{m}, x_{n+1}\right)\right\}=0
\end{aligned}
$$

and

$$
\begin{aligned}
& \limsup _{m, n \rightarrow \infty} M\left(x_{n}, x_{m}\right) \\
& \quad=\limsup _{m, n \rightarrow \infty} \max \left\{\mathcal{D}\left(x_{n}, x_{m}\right), \mathcal{D}\left(x_{n}, x_{n+1}\right), \mathcal{D}\left(x_{m}, x_{m+1}\right), \frac{\mathcal{D}\left(x_{n}, x_{m+1}\right)+\mathcal{D}\left(x_{m}, x_{n+1}\right)}{6 K}\right\}
\end{aligned}
$$




$$
\begin{aligned}
\leq & \limsup _{m, n \rightarrow \infty} \max \left\{\mathcal{D}\left(x_{n}, x_{m}\right), \mathcal{D}\left(x_{n}, x_{n+1}\right), \mathcal{D}\left(x_{m}, x_{m+1}\right),\right. \\
& \left.\frac{K\left(\mathcal{D}\left(x_{n}, x_{m}\right)+\mathcal{D}\left(x_{m}, x_{m+1}\right)\right)+K\left(\mathcal{D}\left(x_{m}, x_{n}\right)+\mathcal{D}\left(x_{n}, x_{n+1}\right)\right)}{6 K}\right\} \\
= & \limsup _{m, n \rightarrow \infty} \mathcal{D}\left(x_{n}, x_{m}\right) \leq \limsup _{m, n \rightarrow \infty} M\left(x_{n}, x_{m}\right) .
\end{aligned}
$$

That is,

$$
\limsup _{m, n \rightarrow \infty} M\left(x_{n}, x_{m}\right)=\limsup _{m, n \rightarrow \infty} \mathcal{D}\left(x_{n}, x_{m}\right) .
$$

Now, by (4.1) we have

$$
\begin{aligned}
\limsup _{m, n \rightarrow \infty} \mathcal{D}\left(x_{n+1}, x_{m+1}\right)= & \limsup _{m, n \rightarrow \infty} \mathcal{D}\left(T x_{n}, T x_{m}\right) \\
\leq & \limsup _{m, n \rightarrow \infty} \mathcal{L}\left(M\left(x_{n}, x_{m}\right)\right) \limsup _{m, n \rightarrow \infty} M\left(x_{n}, x_{m}\right) \\
& +\limsup _{m, n \rightarrow \infty} \mathcal{J}\left(N\left(x_{n}, x_{m}\right)\right) \limsup _{m, n \rightarrow \infty} N\left(x_{n}, x_{m}\right),
\end{aligned}
$$

and so from (4.5) and (4.6) we get

$$
\limsup _{m, n \rightarrow \infty} \mathcal{D}\left(x_{n+1}, x_{m+1}\right) \leq \limsup _{m, n \rightarrow \infty} \mathcal{L}\left(M\left(x_{n}, x_{m}\right)\right) \limsup _{m, n \rightarrow \infty} \mathcal{D}\left(x_{n}, x_{m}\right) .
$$

By $(\mathcal{D} 3)$ we have

$$
\mathcal{D}\left(x_{n}, x_{m}\right) \leq K \mathcal{D}\left(x_{n}, x_{n+1}\right)+K^{2} \mathcal{D}\left(x_{n+1}, x_{m+1}\right)+K^{2} \mathcal{D}\left(x_{m+1}, x_{m}\right) .
$$

Taking limitsup as $n \rightarrow \infty$ in the above inequality, we have

$$
\frac{1}{K^{2}} \limsup _{m, n \rightarrow \infty} \mathcal{D}\left(x_{n}, x_{m}\right) \leq \limsup _{m, n \rightarrow \infty} \mathcal{D}\left(x_{n+1}, x_{m+1}\right) \text {. }
$$

Then by (4.7) we deduce

$$
\frac{1}{K^{2}} \limsup _{m, n \rightarrow \infty} \mathcal{D}\left(x_{n}, x_{m}\right) \leq \limsup _{m, n \rightarrow \infty} \mathcal{L}\left(M\left(x_{n}, x_{m}\right)\right) \limsup _{m, n \rightarrow \infty} \mathcal{D}\left(x_{n}, x_{m}\right) .
$$

Now, since $\lim \sup _{m, n \rightarrow \infty} D\left(x_{n}, x_{m}\right)>0$, then

$$
\frac{1}{K^{2}} \leq \limsup _{m, n \rightarrow \infty} \mathcal{L}\left(M\left(x_{n}, x_{m}\right)\right)
$$

On the other hand, since $\lim \sup _{m, n \rightarrow \infty} \mathcal{L}\left(M\left(x_{n}, x_{m}\right)\right) \leq \frac{1}{K^{2}}$, hence

$$
\limsup _{m, n \rightarrow \infty} \mathcal{L}\left(M\left(x_{n}, x_{m}\right)\right)=\frac{1}{K^{2}}
$$

This implies that

$$
\limsup _{m, n \rightarrow \infty} \mathcal{D}\left(x_{n}, x_{m}\right)=\limsup _{m, n \rightarrow \infty} M\left(x_{n}, x_{m}\right)=0
$$


which is contradiction. Thus, $\limsup _{m, n \rightarrow \infty} \mathcal{D}\left(x_{n}, x_{m}\right)=0$. Now, since $\lim _{m, n \rightarrow \infty} \mathcal{D}\left(x_{n}\right.$, $\left.x_{m}\right)=0$ exists (and is finite), so $\left\{x_{n}\right\}$ is a Cauchy sequence. As $(X, \mathcal{D}, K)$ is a complete $b$ metric-like space, the sequence $\left\{x_{n}\right\}$ in $X$ converges to $z \in X$ so that

$$
\lim _{m, n \rightarrow \infty} \mathcal{D}\left(x_{n}, z\right)=\mathcal{D}(z, z)=\lim _{m, n \rightarrow \infty} \mathcal{D}\left(x_{n}, x_{m}\right)=0 .
$$

From (ii) and (4.1), with $x=x_{n}$ and $y=z$, we obtain

$$
\mathcal{D}\left(x_{n+1}, T z\right)=\mathcal{D}\left(T x_{n}, T z\right) \leq \mathcal{L}\left(M\left(x_{n}, z\right)\right) M\left(x_{n}, z\right)+\mathcal{J}\left(N\left(x_{n}, z\right)\right) N\left(x_{n}, z\right)
$$

On the other hand,

$$
\lim _{n \rightarrow \infty} N\left(x_{n}, z\right)=\lim _{n \rightarrow \infty} \min \left\{\mathcal{D}^{s}\left(x_{n}, x_{n+1}\right), \mathcal{D}^{s}(z, T z), \mathcal{D}^{s}\left(x_{n}, T z\right), \mathcal{D}^{s}\left(z, x_{n+1}\right)\right\}=0
$$

and

$$
\begin{aligned}
\lim _{n \rightarrow \infty} M\left(x_{n}, z\right) & =\lim _{n \rightarrow \infty} \max \left\{\mathcal{D}\left(x_{n}, z\right), \mathcal{D}\left(x_{n}, x_{n+1}\right), \mathcal{D}(z, T z), \frac{\mathcal{D}\left(x_{n}, T z\right)+\mathcal{D}\left(z, x_{n+1}\right)}{6 K}\right\} \\
& \leq \mathcal{D}(z, T z) \quad(\text { by applying Proposition } 2.10(\mathrm{~B})) .
\end{aligned}
$$

Then $\lim _{n \rightarrow \infty} M\left(x_{n}, z\right)=\mathcal{D}(z, T z)$. Again, by using Proposition 2.10(B) and (4.8), we have

$$
\frac{1}{K^{2}} \mathcal{D}(z, T z) \leq \frac{1}{K} \mathcal{D}(z, T z) \leq \lim _{n \rightarrow \infty} \mathcal{D}\left(x_{n+1}, T z\right) \leq \lim _{n \rightarrow \infty} \mathcal{L}\left(M\left(x_{n}, z\right)\right) \mathcal{D}(z, T z)
$$

Now, if $\mathcal{D}(z, T z)>0$, then $\lim _{n \rightarrow \infty} \mathcal{L}\left(M\left(x_{n}, z\right)\right)=\frac{1}{K^{2}}$. This implies

$$
\mathcal{D}(z, T z)=\lim _{n \rightarrow \infty} M\left(x_{n}, z\right)=0,
$$

which is a contradiction. Hence, $\mathcal{D}(z, T z)=0$. That is, $z=T z$.

Example 4.2 Let $X=[0, \infty)$ and $\mathcal{D}: X \times X \rightarrow \mathbb{R}_{+}$be defined by

$$
\mathcal{D}(x, y)=(\max \{x, y\})^{2} .
$$

Clearly, $(X, \mathcal{D}, 2)$ is a complete $b$-metric-like space. Let $T: X \rightarrow X$ be defined by

$$
T x= \begin{cases}\frac{x}{5 \sqrt{1+x^{2}}} & \text { if } x \in[0,1), \\ \frac{\sqrt[4]{x}}{2 \sqrt{1+\sqrt{x}}} & \text { if } x \in(1, \infty) .\end{cases}
$$

Also, define $\mathcal{L}:(0, \infty) \rightarrow\left(0, \frac{1}{4}\right)$ by $\mathcal{L}(t)=\frac{1}{4(1+t)}$. Let $x \preceq y \Leftrightarrow x \leq y$. At first we assume that $x \leq y$. Let $y \in[0,1)$, then $T y \leq \frac{y}{2 \sqrt{1+y^{2}}}$. Also, let $y \in[1, \infty)$, then $T y \leq \frac{y}{2 \sqrt{1+y^{2}}}$. That is, for all $y \in X$, we have

$$
T y \leq \frac{y}{2 \sqrt{1+y^{2}}}
$$


which implies

$$
(T y)^{2} \leq \frac{y^{2}}{4\left(1+y^{2}\right)}
$$

equivalently,

$$
(\max \{T x, T y\})^{2} \leq \frac{(\max \{x, y\})^{2}}{4\left(1+(\max \{x, y\})^{2}\right)},
$$

and so

$$
\mathcal{D}(T x, T y) \leq \frac{\mathcal{D}(x, y)}{4(1+\mathcal{D}(x, y))} \leq \frac{M(x, y)}{4(1+M(x, y))}=\mathcal{L}(M(x, y)) M(x, y) .
$$

Then the conditions of Theorem 4.1 hold and $T$ has a fixed point.

Also we have the following corollaries.

Corollary 4.3 Let $(X, p, \preceq)$ be a partially ordered complete partial metric space, and let $T: X \rightarrow X$ be a non-decreasing mapping such that

$$
p(T x, T y) \leq \mathcal{L}(M(x, y)) M(x, y)+\mathcal{J}(N(x, y)) N(x, y)
$$

for all $x, y \in X$ with $x \preceq y$, where $\mathcal{L} \in \Psi_{\mathcal{L}}^{1}, \mathcal{J}:[0, \infty) \rightarrow[0, \infty)$ is a bounded function and

$$
M(x, y)=\max \left\{p(x, y), p(x, T x), p(y, T y), \frac{p(x, T y)+p(y, T x)}{6}\right\}
$$

and

$$
N(x, y)=\min \left\{p^{s}(x, T x), p^{s}(y, T y), p^{s}(x, T y), p^{s}(y, T x)\right\} .
$$

Also suppose that the following assertions hold:

(i) there exists $x_{0} \in X$ such that $x_{0} \preceq T x_{0}$;

(ii) for an increasing sequence $\left\{x_{n}\right\} \subset X$ converging to $x \in X$, we have $x_{n} \preceq x$ for all $n \in \mathbb{N}$; then $T$ has a fixed point.

Corollary 4.4 Let $(X, d, K, \preceq)$ be a partially ordered complete $b$-metric space, and let $T$ : $X \rightarrow X$ be a non-decreasing mapping such that

$$
d(T x, T y) \leq \mathcal{L}(M(x, y)) M(x, y)+\mathcal{J}(N(x, y)) N(x, y)
$$

for all $x, y \in X$ with $x \preceq y$, where $\mathcal{L} \in \Psi_{\mathcal{L}}^{K}, \mathcal{J}:[0, \infty) \rightarrow[0, \infty)$ is a bounded function and

$$
M(x, y)=\max \left\{d(x, y), d(x, T x), d(y, T y), \frac{d(x, T y)+d(y, T x)}{4 K}\right\}
$$

and

$$
N(x, y)=2 \min \{d(x, T x), d(y, T y), d(x, T y), d(y, T x)\} .
$$


Also suppose that the following assertions hold:

(i) there exists $x_{0} \in X$ such that $x_{0} \preceq T x_{0}$;

(ii) for an increasing sequence $\left\{x_{n}\right\} \subset X$ converging to $x \in X$, we have $x_{n} \preceq x$ for all $n \in \mathbb{N}$; then $T$ has a fixed point.

Remark 4.5 By utilizing the technique of Amini-Harandi [10] and Samet et al. [23], we can obtain corresponding coupled fixed point results from our Theorem 4.1 and Corollaries 4.3 and 4.4 on the basis of the following simple lemma. For more detailed literature on coupled fixed theory, we refer to [24-28].

Lemma 4.6 [23] (A coupled fixed point is a fixed point) Let $F: X \times X \rightarrow X$ be a given mapping. Define the mapping $T: X \times X \rightarrow X \times X$ by

$$
T(x, y)=(F(x, y), F(y, x))
$$

for all $(x, y) \in X \times X$. Then $(x, y)$ is a coupled fixed point of $F$ if and only if $(x, y)$ is a fixed point of $T$.

\section{Fixed point results for cyclic Edelstein-Suzuki contraction}

In 1962, Edelstein [29] proved an important version of the Banach contraction principle. In 2009, Suzuki [30] improved the results of Banach and Edelstein (see also [31, 32]). In recent years, cyclic contraction and cyclic contractive type mapping have appeared in several works (see [33-38]). In this section we first prove the following result, which generalizes corresponding results of Edelstein [29], Suzuki [30] and Kirk et al. [33] to the setting of $b$-metric-like spaces.

Theorem 5.1 Let $(X, \mathcal{D}, K)$ be a complete b-metric-like space, and let $\left\{A_{j}\right\}_{j=1}^{m}$ be a family of nonempty closed subsets of $X$ with $Y=\bigcup_{j=1}^{m} A_{j}$. Let $T: Y \rightarrow Y$ be a map satisfying

$$
T\left(A_{j}\right) \subseteq A_{j+1}, \quad j=1,2, \ldots, m, \text { where } A_{m+1}=A_{1} .
$$

Assume that

$$
\begin{aligned}
\frac{1}{2 K} \mathcal{D}(x, T x) \leq \mathcal{D}(x, y) & \\
\Rightarrow \quad \mathcal{D}(T x, T y) \leq & \frac{\alpha(K+1)}{K} \mathcal{D}(x, y)+\beta[\mathcal{D}(x, T x)+\mathcal{D}(y, T y)] \\
& +\gamma\left[\frac{\mathcal{D}(x, T y)+\mathcal{D}(y, T x)}{3 K}\right]+\delta\left[\frac{\mathcal{D}(x, x)+\mathcal{D}(y, y)}{4 K}\right]
\end{aligned}
$$

for all $x \in A_{i}$ and $y \in A_{i+1}$, where $\alpha, \beta, \gamma, \delta \geq 0$ and $\alpha+\beta+\gamma+\delta<\frac{1}{K+1}$. Then $T$ has a fixed point in $\bigcap_{j=1}^{m} A_{j}$.

Proof Let $x_{0} \in A_{1}$ and define a sequence $\left\{x_{n}\right\}$ in the following way:

$$
x_{n}=T x_{n-1}, \quad n=1,2,3, \ldots .
$$


We have $x_{0} \in A_{1}, x_{1}=T x_{0} \in A_{2}, x_{2}=T x_{1} \in A_{3}, \ldots$ If $x_{n_{0}+1}=x_{n_{0}}$ for some $n_{0} \in \mathbb{N}$, then, clearly, the fixed point of the map $T$ is $x_{n_{0}}$. Hence, we assume that $x_{n} \neq x_{n+1}$ for all $n \in \mathbb{N}$. Clearly, $\frac{1}{2 K} \mathcal{D}\left(x_{n-1}, T x_{n-1}\right) \leq \mathcal{D}\left(x_{n-1}, x_{n}\right)$. Now, from (5.2) we have

$$
\begin{aligned}
\mathcal{D}\left(T x_{n-1}, T x_{n}\right) \leq & \frac{\alpha(K+1)}{K} \mathcal{D}\left(x_{n-1}, x_{n}\right)+\beta\left(\mathcal{D}\left(x_{n-1}, T x_{n-1}\right)+\mathcal{D}\left(x_{n}, T x_{n}\right)\right) \\
& +\gamma\left(\frac{\mathcal{D}\left(x_{n-1}, T x_{n}\right)+\mathcal{D}\left(x_{n}, T x_{n-1}\right)}{3 K}\right)+\delta\left(\frac{\mathcal{D}\left(x_{n-1}, x_{n-1}\right)+\mathcal{D}\left(x_{n}, x_{n}\right)}{4 K}\right),
\end{aligned}
$$

which implies

$$
\begin{aligned}
\mathcal{D}\left(x_{n}, x_{n+1}\right) \leq & \frac{\alpha(K+1)}{K} \mathcal{D}\left(x_{n-1}, x_{n}\right)+\beta\left(\mathcal{D}\left(x_{n-1}, x_{n}\right)+\mathcal{D}\left(x_{n}, x_{n+1}\right)\right) \\
& +\gamma\left(\frac{\mathcal{D}\left(x_{n-1}, x_{n+1}\right)+\mathcal{D}\left(x_{n}, x_{n}\right)}{3 K}\right)+\delta\left(\frac{\mathcal{D}\left(x_{n-1}, x_{n-1}\right)+\mathcal{D}\left(x_{n}, x_{n}\right)}{4 K}\right) .
\end{aligned}
$$

From $(\mathcal{D} 3)$ we have

$$
\mathcal{D}\left(x_{n-1}, x_{n+1}\right) \leq K\left(\mathcal{D}\left(x_{n}, x_{n-1}\right)+\mathcal{D}\left(x_{n}, x_{n+1}\right)\right)
$$

and

$$
\mathcal{D}\left(x_{n}, x_{n}\right) \leq 2 K \mathcal{D}\left(x_{n}, x_{n+1}\right) \leq 2 K\left(\mathcal{D}\left(x_{n}, x_{n-1}\right)+\mathcal{D}\left(x_{n}, x_{n+1}\right)\right)
$$

and so

$$
\frac{\mathcal{D}\left(x_{n-1}, x_{n+1}\right)+\mathcal{D}\left(x_{n}, x_{n}\right)}{3 K} \leq \mathcal{D}\left(x_{n}, x_{n-1}\right)+\mathcal{D}\left(x_{n}, x_{n+1}\right)
$$

Also,

$$
\mathcal{D}\left(x_{n-1}, x_{n-1}\right) \leq 2 K \mathcal{D}\left(x_{n}, x_{n-1}\right) \leq 2 K\left(\mathcal{D}\left(x_{n}, x_{n-1}\right)+\mathcal{D}\left(x_{n}, x_{n+1}\right)\right)
$$

Then

$$
\frac{\mathcal{D}\left(x_{n-1}, x_{n-1}\right)+\mathcal{D}\left(x_{n}, x_{n}\right)}{4 K} \leq \mathcal{D}\left(x_{n}, x_{n-1}\right)+\mathcal{D}\left(x_{n}, x_{n+1}\right) .
$$

Hence, by (5.4), (5.5) and (5.6) we get

$$
\mathcal{D}\left(x_{n}, x_{n+1}\right) \leq \frac{\alpha(K+1)}{K} \mathcal{D}\left(x_{n-1}, x_{n}\right)+(\beta+\gamma+\delta)\left(\mathcal{D}\left(x_{n}, x_{n-1}\right)+\mathcal{D}\left(x_{n}, x_{n+1}\right)\right),
$$

and then

$$
\mathcal{D}\left(x_{n}, x_{n+1}\right) \leq h \mathcal{D}\left(x_{n}, x_{n-1}\right),
$$

where

$$
h=\frac{\left[\frac{\alpha(K+1)}{K}+\beta+\gamma+\delta\right]}{1-(\beta+\gamma+\delta)} .
$$


Now since $(K+1)(\alpha+\beta+\gamma+\delta)<1$, then

$$
\alpha \frac{K+1}{K}+\beta+\gamma+\delta+\frac{1}{K}(\beta+\gamma+\delta)<\frac{1}{K},
$$

which implies

$$
\alpha \frac{K+1}{K}+\beta+\gamma+\delta+<\frac{1}{K}[1-(\beta+\gamma+\delta)] .
$$

Then by Lemma 2.14 we have $\lim _{m, n \rightarrow \infty} \mathcal{D}\left(x_{n}, x_{m}\right)=0$. Now, since $\lim _{m, n \rightarrow \infty} \mathcal{D}\left(x_{n}, x_{m}\right)=0$ exists (and is finite), so $\left\{x_{n}\right\}$ is a Cauchy sequence. Since $(X, \mathcal{D}, K)$ is a complete $b$-metriclike space, the sequence $\left\{x_{n}\right\}$ in $X$ converges to $z \in X$ so that

$$
\lim _{m, n \rightarrow \infty} \mathcal{D}\left(x_{n}, z\right)=\mathcal{D}(z, z)=\lim _{m, n \rightarrow \infty} \mathcal{D}\left(x_{n}, x_{m}\right)=0
$$

It is easy to see that $z \in \bigcap_{j=1}^{m} A_{j}$. Since $x_{0} \in A_{1}$, so the subsequence $\left\{x_{m(n-1)}\right\}_{n=1}^{\infty} \in$ $A_{1}$, the subsequence $\left\{x_{m(n-1)+1}\right\}_{n=1}^{\infty} \in A_{2}$ and, continuing in this way, the subsequence $\left\{x_{m n-1}\right\}_{n=1}^{\infty} \in A_{m}$. All the $m$ subsequences are convergent in the closed sets $A_{j}$, and hence they all converge to the same limit $z \in \bigcap_{j=1}^{m} A_{j}$. Suppose that there exists $n_{0} \in \mathbb{N}$ such that the following inequalities hold:

$$
\frac{1}{2 K} \mathcal{D}\left(x_{n_{0}}, x_{n_{0}+1}\right)>\mathcal{D}\left(x_{n_{0}}, z\right) \quad \text { and } \quad \frac{1}{2 K} \mathcal{D}\left(x_{n_{0}+1}, x_{n_{0}+2}\right)>\mathcal{D}\left(x_{n_{0}+1}, z\right)
$$

Then

$$
\begin{aligned}
\mathcal{D}\left(x_{n_{0}}, x_{n_{0}+1}\right) & \leq K\left(\mathcal{D}\left(x_{n_{0}}, z\right)+\mathcal{D}\left(T x_{n_{0}}, z\right)\right) \\
& <\frac{1}{2} \mathcal{D}\left(x_{n_{0}}, x_{n_{0}+1}\right)+\frac{1}{2} \mathcal{D}\left(x_{n_{0}+1}, x_{n_{0}+2}\right) \\
& <\frac{1}{2} \mathcal{D}\left(x_{n_{0}}, x_{n_{0}+1}\right)+\frac{1}{2} \mathcal{D}\left(x_{n_{0}}, x_{n_{0}+1}\right)=\mathcal{D}\left(x_{n_{0}}, x_{n_{0}+1}\right),
\end{aligned}
$$

which is a contradiction. Hence, for every $n \in \mathbb{N}$, we have

$$
\frac{1}{2 K} \mathcal{D}\left(x_{n}, x_{n+1}\right) \leq \mathcal{D}\left(x_{n}, z\right) \quad \text { or } \quad \frac{1}{2 K} \mathcal{D}\left(x_{n+1}, x_{n+2}\right) \leq \mathcal{D}\left(x_{n+1}, z\right)
$$

and so by (5.2) we have

$$
\begin{aligned}
\mathcal{D}\left(x_{n+1}, T z\right) \leq & \frac{\alpha(K+1)}{K} \mathcal{D}\left(x_{n}, z\right)+\beta\left(\mathcal{D}\left(x_{n}, x_{n+1}\right)+\mathcal{D}(z, T z)\right) \\
& +\gamma\left(\frac{\mathcal{D}\left(x_{n}, T z\right)+\mathcal{D}\left(z, x_{n+1}\right)}{3 K}\right)+\delta\left(\frac{\mathcal{D}\left(x_{n}, x_{n}\right)+\mathcal{D}(z, z)}{4 K}\right)
\end{aligned}
$$

or

$$
\begin{aligned}
\mathcal{D}\left(x_{n+2}, T z\right) \leq & \frac{\alpha(K+1)}{K} \mathcal{D}\left(x_{n+1}, z\right)+\beta\left(\mathcal{D}\left(x_{n+1}, x_{n+2}\right)+\mathcal{D}(z, T z)\right) \\
& +\gamma\left(\frac{\mathcal{D}\left(x_{n+1}, T z\right)+\mathcal{D}\left(z, x_{n+2}\right)}{3 K}\right)+\delta\left(\frac{\mathcal{D}\left(x_{n+1}, x_{n+1}\right)+\mathcal{D}(z, z)}{4 K}\right) .
\end{aligned}
$$


Assume that (5.7) holds. Then, by taking limit as $n \rightarrow \infty$ in (5.7), we get

$$
\lim _{n \rightarrow \infty} \mathcal{D}\left(x_{n+1}, T z\right) \leq \beta \mathcal{D}(z, T z)+\frac{\gamma}{3 K} \lim _{n \rightarrow \infty} \mathcal{D}\left(x_{n}, T z\right)
$$

and hence by Proposition 2.10(B) we have

$$
\frac{1}{K} \mathcal{D}(z, T z) \leq \beta \mathcal{D}(z, T z)+\frac{\gamma}{3} \mathcal{D}(z, T z)
$$

Therefore,

$$
\left(\frac{1}{K}-\beta-\frac{\gamma}{3}\right) \mathcal{D}(z, T z) \leq 0
$$

On the other hand, $\alpha, \beta, \gamma, \delta \geq 0$ and $\alpha+\beta+\gamma+\delta<\frac{1}{K+1}<\frac{1}{K}$. Then $\beta+\frac{\gamma}{3} \leq \beta+\gamma<\frac{1}{K}$. That is, $\frac{1}{K}-\beta-\frac{\gamma}{3}>0$. Hence, $\mathcal{D}(z, T z)=0$, i.e., $z=T z$. If (5.8) holds, then by a similar method, we can deduce that $z=T z$.

If in the above theorem we take $A_{i}=X$ for all $m$, then we deduce the following corollary.

Corollary 5.2 Let $(X, \mathcal{D}, K)$ be a complete $b$-metric-like space, and let $T$ be a self-mapping on X. Assume that

$$
\begin{aligned}
\frac{1}{2 K} \mathcal{D}(x, T x) \leq \mathcal{D}(x, y) & \\
\Rightarrow \quad \mathcal{D}(T x, T y) \leq & \frac{\alpha(K+1)}{K} \mathcal{D}(x, y)+\beta(\mathcal{D}(x, T x)+\mathcal{D}(y, T y)) \\
& +\gamma\left(\frac{\mathcal{D}(x, T y)+\mathcal{D}(y, T x)}{3 K}\right)+\delta\left(\frac{\mathcal{D}(x, x)+\mathcal{D}(y, y)}{4 K}\right)
\end{aligned}
$$

for all $x, y \in X$, where $\alpha, \beta, \gamma, \delta \geq 0$ and $\alpha+\beta+\gamma+\delta<\frac{1}{K+1}$. Then $T$ has a fixed point.

If in Theorem 5.1 we take $\frac{\alpha(K+1)}{K}=\beta=\frac{\gamma}{3 K}=\frac{\delta}{4 K}=R$, then we deduce the following corollary.

Corollary 5.3 Let $(X, \mathcal{D}, K)$ be a complete b-metric-like space, and let $\left\{A_{j}\right\}_{j=1}^{m}$ be a family of nonempty closed subsets of $X$ with $Y=\bigcup_{j=1}^{m} A_{j}$. Let $T: Y \rightarrow Y$ be a map satisfying

$$
T\left(A_{j}\right) \subseteq A_{j+1}, \quad j=1,2, \ldots, m, \text { where } A_{m+1}=A_{1} .
$$

Assume that

$$
\begin{aligned}
\frac{1}{2 K} \mathcal{D}(x, T x) \leq \mathcal{D}(x, y) & \\
\Rightarrow \quad \mathcal{D}(T x, T y) \leq & R[\mathcal{D}(x, y)+\mathcal{D}(x, T x)+\mathcal{D}(y, T y)+\mathcal{D}(x, T y) \\
& +\mathcal{D}(y, T x)+\mathcal{D}(x, x)+\mathcal{D}(y, y)]
\end{aligned}
$$

for all $x \in A_{i}$ and $y \in A_{i+1}$, where $0 \leq R<\frac{1}{(K+1)(7 K+1)+K}$. Then $T$ has a fixed point in $\bigcap_{j=1}^{m} A_{j}$. 
If in Corollary 5.2 we take $\frac{\alpha(K+1)}{K}=\beta=\frac{\gamma}{3 K}=\frac{\delta}{4 K}=R$, then we deduce the following corollary.

Corollary 5.4 Let $(X, \mathcal{D}, K)$ be a complete $b$-metric-like space, and let $T$ be a self-mapping on $X$. Assume that

$$
\begin{aligned}
\frac{1}{2 K} \mathcal{D}(x, T x) \leq \mathcal{D}(x, y) & \\
\Rightarrow \quad \mathcal{D}(T x, T y) \leq & R[\mathcal{D}(x, y)+\mathcal{D}(x, T x)+\mathcal{D}(y, T y)+\mathcal{D}(x, T y) \\
& +\mathcal{D}(y, T x)+\mathcal{D}(x, x)+\mathcal{D}(y, y)]
\end{aligned}
$$

for all $x, y \in X$, where $0 \leq R<\frac{1}{(K+1)(7 K+1)+K}$. Then $T$ has a fixed point.

Corollary 5.5 Let $(X, \sigma)$ be a complete metric-like space, $m \in \mathbb{N}$, let $A_{1}, A_{2}, \ldots, A_{m}$ be nonempty closed subsets of $X$ and $Y=\bigcup_{i=1}^{m} A_{i}$. Suppose that $T: Y \rightarrow Y$ is an operator such that

(i) $Y=\bigcup_{i=1}^{m} A_{i}$ is a cyclic representation of $X$ with respect to $T$;

(ii) Assume that there exists $0 \leq R<\frac{1}{17}$ such that

$$
\frac{1}{2} \int_{0}^{\sigma(x, T x)} \rho(t) d t \leq \int_{0}^{\sigma(x, y)} \rho(t) d t \quad \Longrightarrow \quad \int_{0}^{\sigma(T x, T y)} \rho(t) d t \leq R \int_{0}^{M(x, y)} \rho(t) d t
$$

where

$$
M(x, y)=\sigma(x, y)+\sigma(x, T x)+\sigma(y, T y)+\sigma(x, T y)+\sigma(y, T x)+\sigma(x, x)+\sigma(y, y)
$$

for any $x \in A_{i}, y \in A_{i+1}, i=1,2, \ldots, m$, where $A_{m+1}=A_{1}$, and $\rho:[0, \infty) \rightarrow[0, \infty)$ is a Lebesgue-integrable mapping satisfying $\int_{0}^{\varepsilon} \rho(t) d t>0$ for $\varepsilon>0$. Then $T$ has a fixed point.

Corollary 5.6 Let $(X, \sigma)$ be a complete metric-like space, and let $T: X \rightarrow X$ be a mapping such that for any $x, y \in X$ there exists $0 \leq R<\frac{1}{17}$ such that

$$
\frac{1}{2} \int_{0}^{\sigma(x, T x)} \rho(t) d t \leq \int_{0}^{\sigma(x, y)} \rho(t) d t \Longrightarrow \int_{0}^{\sigma(T x, T y)} \rho(t) d t \leq R \int_{0}^{M(x, y)} \rho(t) d t,
$$

where

$$
M(x, y)=\sigma(x, y)+\sigma(x, T x)+\sigma(y, T y)+\sigma(x, T y)+\sigma(y, T x)+\sigma(x, x)+\sigma(y, y)
$$

and $\rho:[0, \infty) \rightarrow[0, \infty)$ is a Lebesgue-integrable mapping satisfying $\int_{0}^{\varepsilon} \rho(t) d t$ for $\varepsilon>0$. Then $T$ has fixed point.

\section{Application to the existence of solutions of integral equations}

Motivated by the work in [39-41], we study the existence of solutions for nonlinear integral equations using the results proved in the previous section. 
Consider the integral equation

$$
u(t)=\int_{0}^{T} G(t, s) f(s, u(s)) d s \quad \text { for all } t \in[0, T]
$$

where $T>0, f:[0, T] \times \mathbb{R} \rightarrow \mathbb{R}$ and $G:[0, T] \times[0, T] \rightarrow[0, \infty)$ are continuous functions.

Let $X=C([0, T])$ be the set of real continuous functions on $[0, T]$. We endow $X$ with the $b$-metric-like

$$
\mathcal{D}_{\infty}(u, v)=\sup _{t \in[0, T]}(|u(t)|+|v(t)|)^{2} \quad \text { for all } u, v \in X .
$$

Clearly, $\left(X, \mathcal{D}_{\infty}, 2\right)$ is a complete $b$-metric-like space.

Let $(\alpha, \beta) \in X^{2},\left(\alpha_{0}, \beta_{0}\right) \in \mathbb{R}^{2}$ be such that

$$
\alpha_{0} \leq \alpha(t) \leq \beta(t) \leq \beta_{0} \quad \text { for all } t \in[0, T] .
$$

Assume that for all $t \in[0, T]$, we have

$$
\alpha(t) \leq \int_{0}^{T} G(t, s) f(s, \beta(s)) d s
$$

and

$$
\beta(t) \geq \int_{0}^{T} G(t, s) f(s, \alpha(s)) d s
$$

Let, for all $s \in[0, T], f(s, \cdot)$ be a decreasing function, that is,

$$
x, y \in \mathbb{R}, \quad x \geq y \quad \Longrightarrow \quad f(s, x) \leq f(s, y) .
$$

Assume that

$$
\sup _{t \in[0, T]} \int_{0}^{T} G(t, s) d s \leq 1 .
$$

Also, suppose that for all $s \in[0, T]$, for all $x, y \in \mathbb{R}$ with $\left(x \leq \beta_{0}\right.$ and $\left.y \geq \alpha_{0}\right)$ or $\left(x \geq \alpha_{0}\right.$ and $\left.y \leq \beta_{0}\right)$,

$$
\begin{aligned}
|f(s, x)|+|f(s, y)| \leq & \left(\frac{3 \alpha}{2}(x+y)^{2}+\beta\left((x+T x)^{2}+(y+T y)^{2}\right)+\gamma\left(\frac{(x+T y)^{2}+(y+T x)^{2}}{6}\right)\right. \\
& \left.+\delta\left(\frac{(2 x)^{2}+(2 y)^{2}}{8}\right)\right)^{\frac{1}{2}}
\end{aligned}
$$

where $\alpha, \beta, \gamma, \delta \geq 0$ and $\alpha+\beta+\gamma+\delta<\frac{1}{3}$.

Theorem 6.1 Under assumptions (6.2)-(6.7), integral equation (6.1) has a solution in $\{u \in$ $C([0, T]): \alpha \leq u(t) \leq \beta$ for all $t \in[0, T]\}$. 
Proof Define the closed subsets of $X, A_{1}$ and $A_{2}$ by

$$
A_{1}=\{u \in X: u \leq \beta\}
$$

and

$$
A_{2}=\{u \in X: u \geq \alpha\} .
$$

Also define the mapping $T: X \rightarrow X$ by

$$
T u(t)=\int_{0}^{T} G(t, s) f(s, u(s)) d s \quad \text { for all } t \in[0, T] .
$$

Let us prove that

$$
T\left(A_{1}\right) \subseteq A_{2} \quad \text { and } \quad T\left(A_{2}\right) \subseteq A_{1} .
$$

Suppose that $u \in A_{1}$, that is,

$$
u(s) \leq \beta(s) \quad \text { for all } s \in[0, T]
$$

Applying condition (6.5), since $G(t, s) \geq 0$ for all $t, s \in[0, T]$, we obtain that

$$
G(t, s) f(s, u(s)) \geq G(t, s) f(s, \beta(s)) \quad \text { for all } t, s \in[0, T] \text {. }
$$

The above inequality with condition (6.3) imply that

$$
\int_{0}^{T} G(t, s) f(s, u(s)) d s \geq \int_{0}^{T} G(t, s) f(s, \beta(s)) d s \geq \alpha(t)
$$

for all $t \in[0, T]$. Then we have $T u \in A_{2}$.

Similarly, let $u \in A_{2}$, that is,

$$
u(s) \geq \alpha(s) \quad \text { for all } s \in[0, T] .
$$

Using condition (6.5), since $G(t, s) \geq 0$ for all $t, s \in[0, T]$, we obtain that

$$
G(t, s) f(s, u(s)) \leq G(t, s) f(s, \alpha(s)) \quad \text { for all } t, s \in[0, T] \text {. }
$$

The above inequality with condition (6.4) imply that

$$
\int_{0}^{T} G(t, s) f(s, u(s)) d s \leq \int_{0}^{T} G(t, s) f(s, \alpha(s)) d s \leq \beta(t)
$$

for all $t \in[0, T]$. Then we have $T u \in A_{1}$. Also, we deduce that (6.8) holds.

Now, let $(u, v) \in A_{1} \times A_{2}$, that is, for all $t \in[0, T]$,

$$
u(t) \leq \beta(t), \quad v(t) \geq \alpha(t) .
$$


This implies from condition (6.2) that for all $t \in[0, T]$,

$$
u(t) \leq \beta_{0}, \quad v(t) \geq \alpha_{0} .
$$

Now, by conditions (6.6) and (6.7), we have, for all $t \in[0, T]$,

$$
\begin{aligned}
& (|T x|+|T y|)^{2}=\left(\left|\int_{0}^{T} G(t, s) f(s, x(s)) d s\right|+\left|\int_{0}^{T} G(t, s) f(s, y(s)) d s\right|\right)^{2} \\
& \leq\left(\int_{0}^{T} G(t, s)|f(s, x(s))| d s+\int_{0}^{T} G(t, s)|f(s, y(s))| d s\right)^{2} \\
& =\left(\int_{0}^{T} G(t, s)(|f(s, x(s))|+|f(s, y(s))|) d s\right)^{2} \\
& \leq\left(\int _ { 0 } ^ { T } G ( t , s ) \left(\frac{3 \alpha}{2}(x+y)^{2}+\beta\left((x+T x)^{2}+(y+T y)^{2}\right)\right.\right. \\
& \left.\left.+\gamma\left(\frac{(x+T y)^{2}+(y+T x)^{2}}{6}\right)+\delta\left(\frac{(2 x)^{2}+(2 y)^{2}}{8}\right)\right)^{\frac{1}{2}} d s\right)^{2} \\
& \leq\left(\int _ { 0 } ^ { T } G ( t , s ) \left(\frac{3 \alpha}{2}(|x|+|y|)^{2}+\beta\left((|x|+|T x|)^{2}+(|y|+|T y|)^{2}\right)\right.\right. \\
& \left.\left.+\gamma\left(\frac{(|x|+|T y|)^{2}+(|y|+|T x|)^{2}}{6}\right)+\delta\left(\frac{(2|x|)^{2}+(2|y|)^{2}}{8}\right)\right)^{\frac{1}{2}} d s\right)^{2} \\
& \leq\left(\int _ { 0 } ^ { T } G ( t , s ) \left(\frac{3 \alpha}{2} \mathcal{D}_{\infty}(x, y)+\beta\left(\mathcal{D}_{\infty}(x, T x)+\mathcal{D}_{\infty}(y, T y)\right)\right.\right. \\
& \left.\left.+\gamma\left(\frac{\mathcal{D}_{\infty}(x, T y)+\mathcal{D}_{\infty}(y, T x)}{6}\right)+\delta\left(\frac{\mathcal{D}_{\infty}(x, x)+\mathcal{D}_{\infty}(y, y)}{8}\right)\right)^{\frac{1}{2}} d s\right)^{2} \\
& =\frac{3 \alpha}{2} \mathcal{D}_{\infty}(x, y)+\beta\left(\mathcal{D}_{\infty}(x, T x)+\mathcal{D}_{\infty}(y, T y)\right) \\
& +\gamma\left(\frac{\mathcal{D}_{\infty}(x, T y)+\mathcal{D}_{\infty}(y, T x)}{6}\right)+\delta\left(\frac{\mathcal{D}_{\infty}(x, x)+\mathcal{D}_{\infty}(y, y)}{8}\right) \\
& \times\left(\int_{0}^{T} G(t, s) d s\right)^{2} \\
& \leq \frac{3 \alpha}{2} \mathcal{D}_{\infty}(x, y)+\beta\left(\mathcal{D}_{\infty}(x, T x)+\mathcal{D}_{\infty}(y, T y)\right) \\
& +\gamma\left(\frac{\mathcal{D}_{\infty}(x, T y)+\mathcal{D}_{\infty}(y, T x)}{6}\right)+\delta\left(\frac{\mathcal{D}_{\infty}(x, x)+\mathcal{D}_{\infty}(y, y)}{8}\right),
\end{aligned}
$$

which implies

$$
\begin{aligned}
\mathcal{D}_{\infty}(T x, T y) \leq & \frac{3 \alpha}{2} \mathcal{D}_{\infty}(x, y)+\beta\left(\mathcal{D}_{\infty}(x, T x)+\mathcal{D}_{\infty}(y, T y)\right) \\
& +\gamma\left(\frac{\mathcal{D}_{\infty}(x, T y)+\mathcal{D}_{\infty}(y, T x)}{6}\right)+\delta\left(\frac{\mathcal{D}_{\infty}(x, x)+\mathcal{D}_{\infty}(y, y)}{8}\right) .
\end{aligned}
$$

By a similar method, we can show that the above inequality holds if $(u, v) \in A_{2} \times A_{1}$. 
Now, all the conditions of Theorem 5.1 hold and $T$ has a fixed point $z^{*}$ in

$$
A_{1} \cap A_{2}=\{u \in C([0, T]): \alpha \leq u(t) \leq \beta \text { for all } t \in[0, T]\} .
$$

That is, $z^{*} \in A_{1} \cap A_{2}$ is the solution to (6.1).

\section{Competing interests}

The authors declare that they have no competing interests.

\section{Authors' contributions}

All authors contributed equally and significantly in writing this article. All authors read and approved the final manuscript.

\section{Author details}

${ }^{1}$ Department of Mathematics, King Abdulaziz University, P.O. Box 80203, Jeddah, 21589, Saudi Arabia. ${ }^{2}$ Young Researchers and Elite Club, Rasht Branch, Islamic Azad University, Rasht, Iran.

\section{Acknowledgements}

This research was funded by the Deanship of Scientific Research (DSR), King Abdulaziz University, Jeddah, under grant No. (390-130-1433). The first and second authors acknowledge with thanks DSR, KAU for financial support. The authors would like to express their thanks to the referees for their helpful comments and suggestions.

Received: 10 April 2013 Accepted: 2 August 2013 Published: 22 August 2013

\section{References}

1. Matthews, SG: Partial metric topology. In: Proc. 8th Summer Conference on General Topology and Applications. Ann. New York Acad. Sci., vol. 728, pp. 183-197 (1994)

2. Hussain, N, Kadelburg, Z, Radenovic, S, Al-Solamy, FR: Comparison functions and fixed point results in partial metric spaces. Abstr. Appl. Anal. 2012, Article ID 605781 (2012)

3. Bakhtin, IA: The contraction mapping principle in quasimetric spaces. In: Functional Analysis, vol. 30, pp. 26-37/ Ul'yanovsk. Gos. Ped. Inst., Ul'yanovsk (1989)

4. Czerwik, S: Contraction mappings in b-metric spaces. Acta Math. Inform. Univ. Ostrav. 1, 5-11 (1993)

5. Hussain, N, Djorić, D, Kadelburg, Z, Radenović, S: Suzuki-type fixed point results in metric type spaces. Fixed Point Theory Appl. 2012, Article ID 126 (2012)

6. Shah, MH, Simic, S, Hussain, N, Sretenovic, A, Radenovic, S: Common fixed points theorems for occasionally weakly compatible pairs on cone metric type spaces. J. Comput. Anal. Appl. 14, 290-297 (2012)

7. Hussain, N, Shah, MH: KKM mappings in cone b-metric spaces. Comput. Math. Appl. 62, 1677-1684 (2011)

8. Khamsi, MA, Hussain, N: KKM mappings in metric type spaces. Nonlinear Anal. 73, 3123-3129 (2010)

9. Amini Harandi, A: Metric-like spaces, partial metric spaces and fixed points. Fixed Point Theory Appl. 2012, Article ID 204 (2012)

10. Amini-Harandi, A: Fixed point theorems for monotone operators in partially ordered metric-like spaces and application to integral equations. J. Nonlinear Convex Anal. (in press)

11. Aage, CT, Salunke, JN: The results on fixed points in dislocated and dislocated quasi-metric space. Appl. Math. Sci. 2(59), 2941-2948 (2008)

12. Sarma, IR, Kumari, PS: On dislocated metric spaces. Int. J. Math. Arch. 3(1), $72-77$ (2012)

13. Zoto, K, Hoxha, E: Fixed point theorems in dislocated and dislocated quasi-metric spaces. J. Adv. Stud. Topol. 3(4), 119-124 (2012)

14. Aage, CT, Salunke, JN: Some fixed point theorems for expansion onto mappings on cone metric spaces. Acta Math. Sin. Engl. Ser. 27(6), 1101-1106 (2011)

15. Kumar, S, Garg, SK: Expansion mapping theorems in metric spaces. Int. J. Contemp. Math. Sci. 4(36), 1749-1758 (2009)

16. Kumar, S: Common fixed points theorems for expansion mappings in various spaces. Acta Math. Hung. 118(1-2), 9-28 (2008)

17. Daffer, ZP, Kaneko, H: On expansive mappings. Math. Jpn. 7, 733-735 (1992)

18. Rhoades, BE: An expansion mapping theorem. Jnanabha 23, 151-152 (1993)

19. Wang, SZ, Li, BY, Gao, ZM, Iseki, K: Some fixed point theorems on expansion mappings. Math. Jpn. 29, 631-636 (1984)

20. Xiang, T, Yuan, R: A class of expansive-type Krasnosel'skii fixed point theorems. Nonlinear Anal. 71, $3229-3239$ (2009)

21. Han, $Y, X u, S$ : Some new theorems of expanding mappings without continuity in cone metric spaces. Fixed Point Theory Appl. 2013, Article ID 3 (2013)

22. Agarwal, RP, El-Gebeily, MA, O’Regan, D: Generalized contractions in partially ordered metric spaces. Appl. Anal. 87, 1-8 (2008)

23. Samet, B, Vetro, C, Vetro, P: Fixed point theorem for $\alpha$ - $\psi$ contractive type mappings. Nonlinear Anal. 75, 2154-2165 (2012)

24. Yeol, JC, Shah, MH, Hussain, N: Coupled fixed points of weakly F-contractive mappings in topological spaces. Appl. Math. Lett. 24, 1185-1190 (2011)

25. Gordji, ME, Akbartabar, E, Cho, YJ, Ramezani, M: Coupled common fixed point theorems for mixed weakly monotone mappings in partially ordered metric spaces. Fixed Point Theory Appl. 2012, Article ID 95 (2012)

26. Hussain, N, Shah, MH, Kutbi, MA: Coupled coincidence point theorems for nonlinear contractions in partially ordered quasi-metric spaces with a Q-function. Fixed Point Theory Appl. 2011, Article ID 703938 (2011) 
27. Sintunavarat, W, Cho, YJ, Kumam, P: Coupled fixed point theorems for contraction mapping induced by cone ball-metric in partially ordered spaces. Fixed Point Theory Appl. 2012, Article ID 128 (2012)

28. Berinde, $\mathrm{V}$ : Generalized coupled fixed point theorems for mixed monotone mappings in partially ordered metric spaces. Nonlinear Anal. 74, 7347-7355 (2011)

29. Edelstein, M: On fixed and periodic points under contractive mappings. J. Lond. Math. Soc. 37, 74-79 (1962)

30. Suzuki, T: A new type of fixed point theorem in metric spaces. Nonlinear Anal. 71(11), 5313-5317 (2009)

31. Salimi, P, Karapinar, E: Suzuki-Edelstein type contractions via auxiliary functions. Math. Probl. Eng. 2013, Article ID 648528 (2013)

32. Suzuki, T: A generalized Banach contraction principle that characterizes metric completeness. Proc. Am. Math. Soc. 136, 1861-1869 (2008)

33. Kirk, WA, Srinavasan, PS, Veeramani, P: Fixed points for mapping satisfying cyclical contractive conditions. Fixed Point Theory 4, 79-89 (2003)

34. Păcurar, M, Rus, IA: Fixed point theory for cyclic $\varphi$-contractions. Nonlinear Anal. 72(3-4), 1181-1187 (2010)

35. Petruşel, G: Cyclic representations and periodic points. Stud. Univ. Babeş-Bolyai, Math. 50, 107-112 (2005)

36. Aydi, H, Vetro, C, Sintunavarat, W, Kumam, P: Coincidence and fixed points for contractions and cyclical contractions in partial metric spaces. Fixed Point Theory Appl. 2012, Article ID 124 (2012)

37. Sintunavarat, W, Kumam, P: Common fixed point theorem for cyclic generalized multi-valued contraction mappings. Appl. Math. Lett. 25(11), 1849-1855 (2012)

38. Nashine, HK, Sintunavarat, W, Kumam, P: Cyclic generalized contractions and fixed point results with applications to an integral equation. Fixed Point Theory Appl. 2012, Article ID 217 (2012)

39. Agarwal, RP, Hussain, N, Taoudi, MA: Fixed point theorems in ordered Banach spaces and applications to nonlinear integral equations. Abstr. Appl. Anal. 2012, Article ID 245872 (2012)

40. Hussain, N, Khan, AR, Agarwal, RP: Krasnosel'skii and Ky Fan type fixed point theorems in ordered Banach spaces. J. Nonlinear Convex Anal. 11(3), 475-489 (2010)

41. Parvaneh, V, Roshan, JR, Radenović, S: Existence of tripled coincidence point in ordered b-metric spaces and application to a system of integral equations. Fixed Point Theory Appl. 2013, Article ID 130 (2013)

doi:10.1186/1029-242X-2013-402

Cite this article as: Alghamdi et al.: Fixed point and coupled fixed point theorems on $b$-metric-like spaces. Journal of Inequalities and Applications 2013 2013:402.

\section{Submit your manuscript to a SpringerOpen ${ }^{\ominus}$ journal and benefit from:}

- Convenient online submission

- Rigorous peer review

- Immediate publication on acceptance

- Open access: articles freely available online

- High visibility within the field

- Retaining the copyright to your article 